\title{
Perioperative goal-directed fluid therapy related reduction in postoperative epidural $0.1 \%$ Bupivacaine consumption using PCEA infusion in total hip arthroplasty patients
}

\begin{abstract}
$\underline{\text { A. Andrijauskas }}{ }^{1}$, S. Svediene ${ }^{1}$, G. Kvederas ${ }^{2}$, N. Porvaneckas ${ }^{3}$, P. Andrijauskas ${ }^{4}$, A. Valiulyte ${ }^{4}$ 1 Vilnius University, Faculty of Medicine, Institute of Clinical Medicine, Clinic of Anesthesiology and Intensive Care - Vilnius (Lithuania), 2 Vilnius University, Faculty of Medicine, Institute of Clinical Medicine,Clinic of Rheumatology, Orthopaedics Traumatology and Reconstructive Surgery - Vilnius (Lithuania), 3 Vilnius University, Faculty of Medicine, Institute of Clinical Medicine, Clinic of Rheumatology, Orthopaedics Traumatology and Reconstructive Surgery - Vilnius (Lithuania), 4 Vilnius University, Faculty of Medicine, Institute of Clinical Medicine, Clinic of Anaesthesiology and Intensive Care - Vilnius (Lithuania).
\end{abstract}

Background and Goal of Study: Impact of perioperative goal-directed fluid administration on postoperative consumption of analgesics is not clear. Study protocol was part of a bigger RCT* .

Materials and Methods: Intervention group patients $(n=39)$ undergoing THA received perioperative goal directed fluid management guided by haemodynamic and microcirculation related parameters using mVLT method* . Controls $(n=40)$ received non-goal-directed fluid management. All subjects received $24 \mathrm{~h}$ postoperative patient controlled epidural analgesia (PCEA) with $0.1 \%$ Bupivacaine. Basal infusion was $5 \mathrm{ml} / \mathrm{h}$ and $5 \mathrm{ml}$ rescue boluses were followed by 30 min refractory period.

\section{Results and Discussion: Pain scores}

and patient satisfaction were similar in both groups, but mean hourly and total 24-h consumption of bupivacaine (mg) in intervention group was lower than in controls: $6.637+/-1.51$ vs.

$7.591+/-1.64(\mathrm{p}=0.01)$, and

$133.811+/-33.13$ vs. $151.661+/-32.03$ $(p=0.02)$, respectively. We speculate that optimised fluid status may have reduced the stress response to surgery and therefore pain was less pronounced in the intervention group. Conclusion(s): Postoperative pain management in patients who received goal-directed fluid administration was achieved with lesser dose of epidural

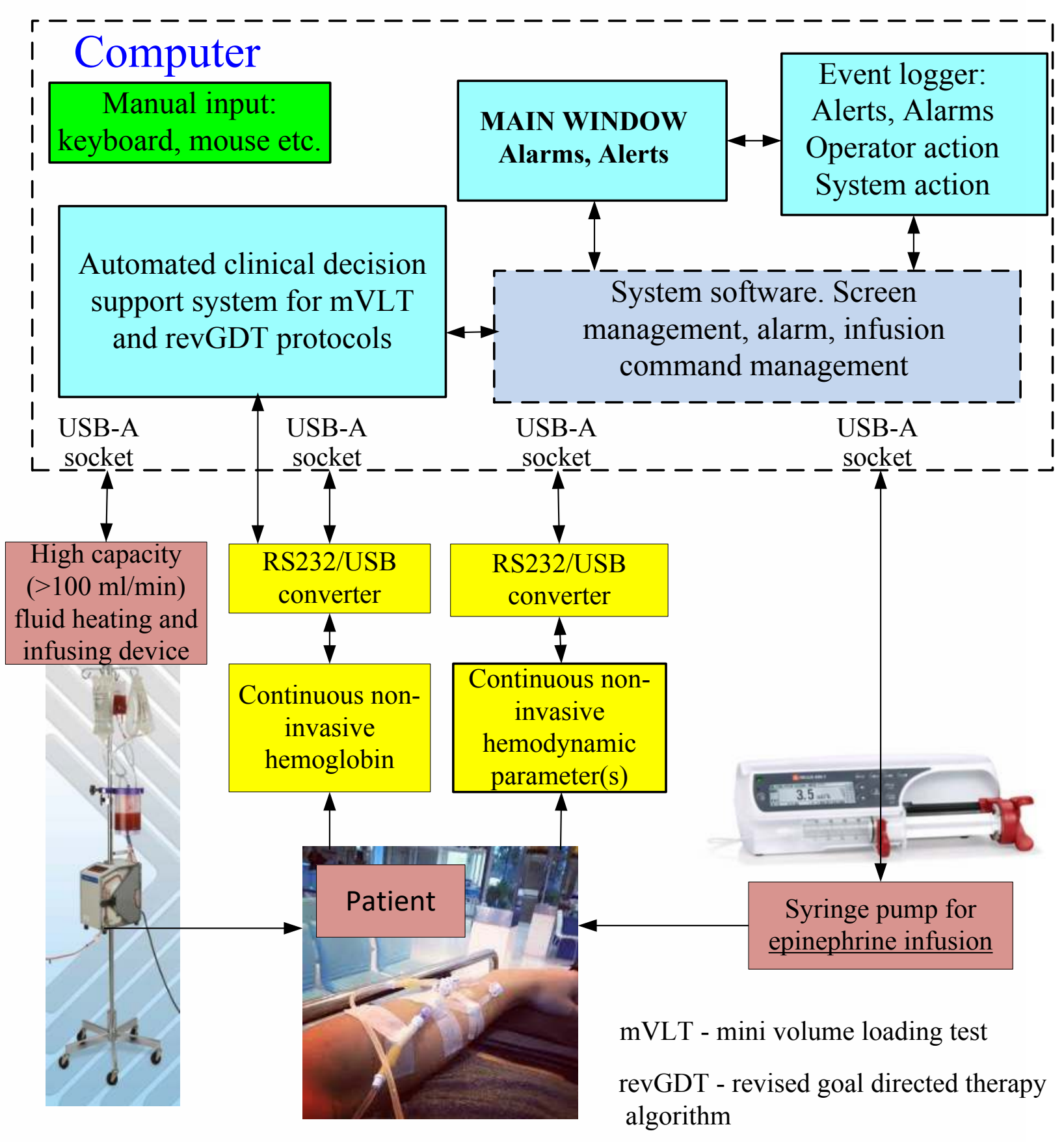
bupivacaine. 